\title{
Earthquake Response and Psychosocial Health Outcomes: Applying Lessons From Integrating Systems of Care and Recovery to Haiti
}

\author{
Carol S. Fullerton, PhD; Dori B. Reissman, MD, MPH; Christine Gray, MPH;
} Brian W. Flynn, EdD; Robert J. Ursano, MD

$\mathrm{O}$ n January 12, 2010, an earthquake measuring 7.0 on the Richter scale struck Haiti, affecting approximately 3.5 million people. As of this writing, information about casualty figures is provisional; however, reports indicate potentially more than 100000 have died, and an even greater number are homeless with destruction of critical infrastructure, including basic utilities, transportation, communication, and health. ${ }^{1}$ The psychological consequences of this event will be significant, and perhaps by examining previous disasters we can apply evidence-based interventions to ensure a comprehensive and integrated response to Haiti.

In this issue of Disaster Medicine and Public Health Preparedness, Cairo et $\mathrm{al}^{2}$ describe posttraumatic stress disorder (PTSD) among residents returning to the city of Pisco, Peru, 5 months after a level 8.0 earthquake struck in 2007. The authors report 25.2\% of their study population endorsed symptoms consistent with a diagnosis of PTSD, which was strongly associated with loss of one's church, inadequate food or shelter, loss of job, poor social support, and personal injury. PTSD is one of the behavioral and mental health outcomes that require careful assessment and attention by planners and decision-making authorities to properly target resources that can influence the trajectory of health and community reconstruction for affected people. The authors' findings highlight the need to better understand the range of behavioral health challenges occurring in the aftermath of unanticipated, catastrophic natural disasters. The public health approach to the behavioral, mental, and psychosocial health needs of disaster populations includes a needs assessment of individual people, community resources, and systems that establish and maintain collaboration across disciplines, involvement of community leaders, and integration of mental and behavioral health strategies with health and recovery plans. An integrated approach is critical to preparedness and response in order to develop interventions that positively impact psychosocial health outcomes following natural disasters such as the earthquakes in Haiti and Peru. ${ }^{3}$

Much disaster research, such as the study by Cairo et al, ${ }^{2}$ focuses on PTSD as a recognized and well-defined result of exposure to traumatic events that threaten life. However, it is important to remember that PTSD is only one of the behavioral and mental health consequences of exposure to trauma and disaster. ${ }^{4-6}$ People exposed to traumatic events are at increased risk for depression, anxiety, and phobic disorders; health risk behaviors such as increased use of caffeine, tobacco, alcohol, prescription medicine, and illicit substances; and exacerba- tion of underlying chronic illness through loss of health care resources. ${ }^{6,7}$ Outcomes are affected by the social, cultural, and spiritual context of the disaster community; the biological makeup of the people affected; and their past experiences and expectations regarding such events. Hostility, with its accompanying social disruption, feelings of frustration, and perception of chaos, is common following large-scale community disasters. ${ }^{6}$ These human factors interact with the intensity and duration of disaster exposure and the availability and equitable distribution of critical resources (eg, food, water, clothing, shelter, and medical and psychosocial support) to produce transient distress or more enduring and sometimes debilitating health outcomes.

There is a growing consensus among disaster mental health experts about the use of psychological first aid ${ }^{8}$ for early intervention. Models of psychological first aid incorporate scientifically supported elements that include a sense of safety, calm, hope, efficacy (ability to do things), and connectedness to others. Overall, the goal of psychological first aid is to foster effective coping through compassionate contact, reunification with loved ones or other social supports, and concrete problem solving (eg, securing resources to meet basic needs, managing disputes, and taking steps to recoup losses). For example, the ability of displaced families to find shelter with family members is typically seen as a preferable alternative to other options. However, seldom discussed in the literature is the increased exposure and increased stress over time that often accompanies these new living patterns. A number of practical psychological first aid models are emerging but require empirical evaluation, ${ }^{9}$ especially with respect to cross-cultural application.

The extensive and community-wide bereavement and grief of disaster pose special challenges. ${ }^{10-12}$ Although the death of loved ones is always painful, an unexpected and violent death is most difficult and more so when there are multiple losses. Destruction of culturally significant places may be associated with communal grief that can be related to elevated rates of depression in the aftermath of disasters, as was seen in Nicaraguan adolescents after Hurricane Mitch. ${ }^{13}$ The experience of loss and grief is exacerbated when conditions do not allow for customary death and burial rituals that facilitate the grieving process. Effective leadership after disasters includes grief leadership, ie, giving permission and teaching and showing people how to grieve. ${ }^{14}$ In community disasters, leaders and responders and their families are directly affected by the disaster. Leaders and responders are also exposed to the traumatic event and its con- 
sequences. Without effective stress management programs, they may also become casualties, with resulting loss of their ability to effectively serve people in need.

Mental health intervention in disaster requires more than preventing or treating a disorder. Providing comfort to bereaved people, helping survivors organize recovery tasks, helping identify and managing stress, and reducing family separation are critical interventions. The practical evidence about disaster interventions, especially early interventions, is far less robust than anyone would hope. ${ }^{8,15}$ Broadly speaking, interventions that may impact the trajectory of recovery span the gamut from population-based strategies such as mass communication, legal remedies, government policy, leadership consultation, and programmed services to individualized clinical techniques in a treatment environment. ${ }^{7}$ From a systems standpoint, treatment options must be tailored to people who require specific levels of care. While challenging, system efficiencies are achieved when a real-time inventory or needs assessment of mental health and other related resources is maintained and used to optimize appropriate and smooth referrals.

Understanding the historical and cultural experience of a disaster community is critical to intervention planning. Culture and faith influence how disaster populations view the disaster, how reactions are expressed, how the effects are interpreted, what is considered helpful, and what it takes to become whole again. ${ }^{16,17}$ In the present Haiti earthquake, the people of Haiti have suffered long-standing economic and health deprivation; unfulfilled expectations of foreign governments and nongovernmental organizations; government repression, corruption, and instability; and the concentration of wealth and power in the hands of a minute percentage of the population. At the same time, one consistently hears about the resilience of the Haitian people in the face of severe and long-standing adversity. It is important to understand the nature and range of religious beliefs and the postdisaster capacity of the existing faith community leaders and structures. In many places such as Haiti, mixed religious traditions exist (eg, Voodoo, Santeria, and Catholicism). These traditions can be confusing to outside helpers who do not understand the complexities or who may attempt to institute changes in religious beliefs. ${ }^{18}$ Although help from religious groups is typically central to disaster recovery around the world, care must be taken to understand and respect religious differences and avoid proselytizing that can add additional stress to already highly impacted victims and survivors.

Colocation and integration of mental health services with medical and social services generally leads to a more comprehensive system of care. ${ }^{7}$ Integrated systems of care are more likely to meet the complex needs of disaster-impacted people such as needs for information; medical and mental health care services; and legal, faith-based, and social services (eg, housing, food and water, transportation, vocational counseling, and de- pendent care). This integration of services facilitates engagement with mental health professionals to assist with future evaluation and treatment needs.

Integrating mental and behavioral health into command, control, and long-term recovery efforts in medical care and public health reduces stigma and increases opportunities to promote psychological health. Working with leaders to understand and anticipate shifting needs and resources can help manage expectations and promote calm. Promoting the resilience of disaster workers and responders through education on safety and stress management, as well as requiring appropriate shift and rest cycles, can improve functioning and reduce burnout and compassion fatigue. ${ }^{19}$ Coordinating communications and information such that messages are clear, culturally sensitive, and consistent helps reduce chaos and confusion, which increase distress and the risk of disease and injury. ${ }^{18}$ Integrating these multifaceted approaches can have a cascading effect that improves psychological health outcomes.

As behavioral health providers from multiple perspectives, traditions, and nations converge on an area in the wake of disaster, conflict and competition among them is likely. For this reason, it is important to establish as much command, control, and management over the resources as is politically, legally, and administratively possible. That authority must be well versed on the state of the science, available and sustainable resources, and practice in this area. This process requires close collaboration with community leaders to understand the local landscape and to ensure the efforts of disaster workers are supportive rather than imposing.

Managing expectations in the aftermath of a major disaster is a significant leadership challenge. Individual and collective histories, as well as current experience, shape expectations. In the case of Haiti, people who expected a more robust response in the early hours and days may have had their expectations of their surroundings changed forever. On the other hand, as the health and medical response becomes more robust in terms of resources, access to care, quality of care, and meeting other needs, expectations for the future may build. From a community perspective, it is important to avoid building expectations of ongoing intervention and involvement that cannot be sustained.

It is entirely conceivable that in the months to come, Haitians may experience a reasonable level of public health, behavioral health, and medical support. Careful policy balances must be considered to provide optimal care while avoiding expectations of care, attention, and resources that are not sustainable over time. After every disaster, eventually the cameras leave, the helpers leave, and attention wanes. What remains are people left to live and cope with very changed lives. Careful and sensitive policy determinations have a major role in determining the nature and direction of those changes. 


\section{About the Authors}

Author Affiliations: Department of Psychiatry, Center for the Study of Traumatic Stress, Uniformed Services University of the Health Sciences, Bethesda, Maryland (Dr Fullerton, Dr Reissman, Ms Gray, Dr Flynn, and Dr Ursano); US Public Health Service, Rockville, Maryland (Dr Reissman); and National Institute for Occupational Safety and Health, Centers for Disease Control and Prevention, Atlanta, Georgia (Dr Reissman).

Correspondence: Carol S. Fullerton, PhD (cfullert@erols.com), Department of Psychiatry, Uniformed Services University of the Health Sciences, 4301 Jones Bridge Road, Bethesda, Maryland 20814.

Disclaimer: The opinions expressed in this article are those of the authors and, therefore, do not necessarily reflect the views of the Department of Defense, the Uniformed Services University of the Health Sciences, the US Public Health Service, the Center for the Study of Traumatic Stress, or the National Institute for Occupational Safety and Health, or the Centers for Disease Control and Prevention.

\section{REFERENCES}

1. World Health Organization. Public Health Risk Assessment and Interventions: Earthquake: Haiti. Geneva, Switzerland: World Health Organization; 2010. WHO reference No. WHO/HSE/GAR/DCE/2010.1.

2. Cairo JB, Dutta S, Nawaz H, Hashmi S, Kasl S, Bellido E. The prevalence of posttraumatic stress disorder among adult earthquake survivors in Peru. Disaster Med Public Health Prep. 2010;4(1):39-46.

3. Landesman LY. Public Health Management of Disasters: The Practice Guide. Washington, DC: American Public Health Association; 2005.

4. Galea S, Brewin CR, Gruber M, et al. Exposure to hurricane-related stressors and mental illness after Hurricane Katrina. Arch Gen Psychiatry. 2007; 64(12):1427-1434.

5. Norris FH, Friedman MJ, Watson PJ, Byrne CM, Diaz E, Kaniasty K. 60,000 disaster victims speak, part I: an empirical review of the empirical literature: 1981-2001. Psychiatry. 2002;65(3):207-239.

6. Ursano RJ, Fullerton CS, Weisaeth L, Raphael B, eds. Textbook of Disaster Psychiatry. Cambridge, England: Cambridge University Press; 2007.

7. Reissman DB, Schreiber MD, Shultz JM, Ursano RJ. Disaster mental and behavioral health. In: Koenig KL, Schultz CH, eds. Disaster Medicine. Cambridge, England: Cambridge University Press; 2010:103-112.

8. Hobfoll SE, Watson PJ, Bell CC, et al. Five essential elements of imme- diate and mid-term mass trauma intervention: empirical evidence. Psychiatry. 2007;70(4):283-315.

9. Watson PJ, Ruzek JI. Academic/state/federal collaborations and the improvement of practices in disaster mental health services and evaluation. Adm Policy Mental Health. 2009;36(3):215-220.

10. Fullerton CS, Ursano RJ, Kao TC, Bharitya VR. Disaster-related bereavement: acute symptoms and subsequent depression. Aviat Space Environ Med. 1999;70(9):902-909.

11. Raphael B, Martinek N, Wooding S. Assessing traumatic bereavement. In: Wilson JP, Keane TM, eds. Assessing Psychological Trauma and PTSD. 2nd ed. New York, NY: Guilford Press; 2004:492-510.

12. Shear K, Frank E, Houck PR, Reynolds CF III. Treatment of complicated grief: a randomized controlled trial. JAMA. 2005;293(21):2601-2608.

13. Goenjian AK, Molina L, Steinberg AM, et al. Posttraumatic stress and depressive reactions among Nicaraguan adolescents after Hurricane Mitch. Am J Psychiatry. 2001;158(5):788-794.

14. Ursano RJ, Fullerton CS. Cognitive and behavioral responses to trauma. J Appl Soc Psychol. 1990;20(21):1766-1775.

15. Watson P. Early intervention for trauma-related problems following mass trauma. In: Ursano RJ, Fullerton CS, Weisaeth L, Raphael B, eds. Textbook of Disaster Psychiatry. Cambridge, England: Cambridge University Press; 2007:121-139.

16. Department of Health and Human Services. Mental Health: Culture, Race, and Ethnicity: A Supplement to Mental Health: A Report of the Surgeon General. Rockville, MD: US Department of Health and Human Services, Substance Abuse and Mental Health Services Administration, Center for Mental Health Services; 2001. DHHS publication SMA-01-3613.

17. Department of Health and Human Services. Developing Cultural Competence in Disaster Mental Health Programs: Guiding Principles and Recommendations. Rockville, MD: US Department of Health and Human Services, Substance Abuse and Mental Health Services Administration, Center for Mental Health Services; 2003. DHHS publication SMA 3828

18. Center for the Study of Traumatic Stress. Earthquake. Center for the Study of Traumatic Stress Web site. http://cstsonline.com/resources/category -35_earthquake. Accessed January 29, 2010.

19. Flynn B, Lane CF. Integrating organizational and behavioral health principles to promote resilience in extreme events. In: Cooper C, Burke R, eds. International Terrorism and Threats to Security: Managerial and Organizational Challenges. Northhampton, MA: Edward Elgar Publishing; 2008: $185-218$. 